\title{
Polyelectrolyte-coated carbons used in the generation of blue energy from salinity differences
}

\author{
Silvia Ahualli, ${ }^{* a}$ M.L. Jiménez, ${ }^{a}$ María M. Fernández, ${ }^{a}$ Guillermo Iglesias, ${ }^{a}$ Doriano Brogioli, ${ }^{b}$ and \\ Ángel V. Delgado, ${ }^{a}$
}

Received Xth $X X X X X X X X X X 20 X X$, Accepted Xth $X X X X X X X X X 20 X X$

First published on the web Xth $X X X X X X X X X X 20 X X$

DOI: $10.1039 / b 000000 x$

In this work we present a method for the production of clean, renewable electrical energy from the exchange of solutions with different salinities. Activated carbon films are coated with negatively or positively charged polyelectrolytes by well-established adsorption methods. When two oppositely charged coated films are placed in contact with an ionic solution, the potential difference between them will be equal to the difference between their Donnan potentials, and hence, energy can be extracted by building an electrochemical cell with such electrodes. A model is elaborated on the operation of the cell, based on the electrokinetic theory of soft particles. All the features of the model are experimentally reproduced, although a small quantitative difference concerning the maximum opencircuit voltage is found, suggesting that the coating is the key point to improve the efficiency. In the used experimental conditions, we obtain a power of $12.1 \mathrm{~mW} / \mathrm{m}^{2}$. Overall, the method proves to be a fruitful and simple approach to salinity-gradient energy production.

Obtaining energy from salinity differences as those existing in river mouths ${ }^{1,2}$ is a challenging task involving various complications. Although several attempts have been reported, ${ }^{3-10}$ no one appears to prevail over the others, and a final choice has not been made. This applies as well to the recent proposals of the capmix group, ${ }^{11}$ based on the change in the capacitance of the electrical double layer (EDL) at the interface between a conducting electrode and an ionic solution, when the salinity of the latter is modified.

The capmix methods are not the only ones devised to harvest salinity difference energy. Desalination techniques operated in reverse are good candidates, in particular, pressureretarded osmosis (PRO), ${ }^{8,12,13}$ and reverse electrodyalisis (RED). ${ }^{3,14}$ In the former, fresh water is allowed to flow through a semipermeable membrane into a pressurized sea

${ }^{a}$ Department of Applied Physics, School of Sciences, University of Granada, 18071, Granada, Spain.

${ }^{b}$ Dipartimento di Scienze della Salute, Universit degli Studi di MilanoBicocca, Via Cadore 48, Monza (MB) 20900, Italy.

*Corresponding author, E-mail: sahualli@ugr.es water chamber; this high-pressure solution is used to obtain electrical energy by depressurizing it through a turbine. In RED, concentrated salt solutions and fresh water flow through alternating cells which are separated by ion exchange membranes; the cells will be alternatively enriched in cations and anions, thus producing, respectively, negative and positive potentials at the corresponding electrodes. If the number of cells connected is large enough, the total voltage will be larger than the electrode reaction potential, and energy can be extracted. Although considerable advances have been produced in both techniques, ${ }^{4-7,9,15}$ they are mostly at the laboratory scale. However, a recent analysis has shown that, at least for the river and sea water availabilities in the Dutch coast, a 1 MW RED plant could be competitive in the near future. ${ }^{16}$

Recent approaches focus on a better understanding of the power generation mechanisms using single synthetic nanopores. ${ }^{7,17,18}$ In these cases, ion selectivity is associated to preferential adsorption and transport of counterions over coions in the channel. Finally, the so-called mixing entropy battery (MEB) method has been described as a promising technology in which battery electrodes store or release specifically adsorbed ions when the salinity of the solution in contact with them is changed. ${ }^{10,19}$

Regarding capmix technologies, two main approaches can be identified, depending on the origin of the voltage difference between the electrodes. In one of them, called Capacitive energy extraction from Double Layer Expansion (CDLE), the electrodes are charged with an external source in presence of a salty solution. This produces an EDL of high capacitance, which is subsequently discharged in presence of fresh solution, that is, at lower capacitance and hence, larger voltage. A net energy is gained because charge is returned to the external circuit at a higher potential than that at which it was placed on the electrodes. ${ }^{20-23}$ Despite the advantage of using cheap activated carbon particles, this method has the problem of leakage, and hence, the gained energy not always compensates for the energy invested in charging the electrodes. ${ }^{24}$

A different approach is that of the Capacitive energy extraction by Donnan Potential (CDP). In this case, the method

This journal is @ The Royal Society of Chemistry [year]

Faraday Discuss., [year], [vol], 1-7 | 1 
is self-sufficient (meaning that it works without the need of external power sources), since the origin of the voltage difference is the Donnan potential of the membranes separating the solution from the carbon particles. Placing one electrode in contact with a cationic membrane and the other with an anionic one produces a voltage difference between both electrodes. This method relies on the change of the Donnan potential with the salinity of the solution: the potential difference increases when fresh or river water enters the inter-electrode spacer, and decreases when sea water gets in. If an external load is connected to the electrodes, current will flow through it in both steps, although in opposite directions. Hence, energy will be obtained every time the water is exchanged. Since no external source is necessary, leakage is a secondary problem and very promising results have been already obtained. ${ }^{25,26}$ In this case, the most important challenges come from the cost and fouling and bio-fouling problems that prevent a long use of the membranes. Recently, Hatzell et al. ${ }^{27}$ described a significant improvement on the CDP technique by immersing the electrodes in a so-called bioelectrochemical system (BES), such as a microbial fuel cell, which favors the charging of the electrodes. All the techniques described require forcing the solutions through the cell, whatever the design. This means that pumping energy or hydraulic losses should be considered when establishing the net power achievable with the device. Only for large scale setups can we expect that the obtained energy outbalances the pumping energy. At the small scale of our experiments, this is not possible.

In this work we propose a method that takes advantage of the two capmix procedures described. In Fig. 1 we represent its principles. We begin by considering two soft conductive particles. By soft particles we mean those formed by a rigid core and a permeable polyelectrolyte layer as schematically represented in the figure. In our case, particles are made of a conductive (activated carbon) core and a polyelectrolyte layer, cationic in the case of the left particle and anionic on the right particle, immersed in a $500 \mathrm{mM} \mathrm{NaCl}$ solution that intends to simulate sea water. In Fig. $1 \mathrm{~b}$ it is represented the potential profile $\Psi$ with respect to the bulk solution. The voltage difference between particles is $V=\Psi_{S}(+)-\Psi_{S}(-)$, where the subscript $S$ indicates the surface properties, and the sign $+(-)$ indicate the particle with the cationic (anionic) polyelectrolyte shell.

At the initial stage (stage 0), the cores are not initialled charged and they are in presence of a salty solution. We restrict ourselves to the case that the polyelectrolyte layers are thick enough for the Donnan potential $\Delta \Psi_{D}^{ \pm}$(salty) to be established (positive in one layer, negative in the other) and the potential profile of Fig. 1b-stage 0 is generated. For the sake of clarity, we are considering an antisymmetric situation of the polymer charge density, and this leads to $\Delta \Psi_{D}^{+}=-\Delta \Psi_{D}^{-}$. Since the particles are not charged, no electric double layer

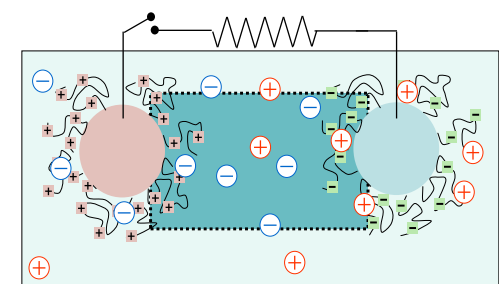

a)

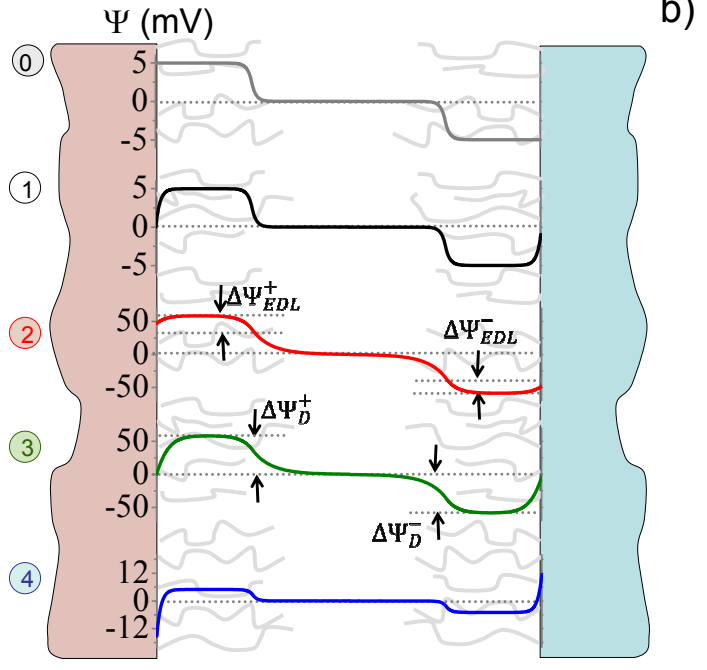

Fig. 1 a) Schematic representation of two carbon particles coated by a cationic polyelectrolyte (left particle) and an anionic one (right particle) immersed in an electrolyte solution and externally connected through an electric circuit. b) Equilibrium electric potential profiles (see text) with respect to the bulk solution between the surfaces of both particles after the different steps of the cycle. 0 : particles in salty solution, open circuit; 1 : particles connected; 2 : open circuit, fresh water; 3: particles connected; 4: open circuit, salty water. Note the different scales of the ordinate axes in stages 0 , 1 and 4.

(EDL) is formed on the particle surface and the potential profile is flat. As a consequence, the surface potential $\Psi_{S}$ with respect to the bulk solution is $\Psi_{S}^{ \pm}=\Delta \Psi_{D}^{ \pm}$(salty), and the potential difference between both particles is $V=\Delta \Psi_{D}^{+}$(salty) $\Delta \Psi_{D}^{-}$(salty) $=2 \Delta \Psi_{D}^{+}$(salty).

If we externally connect both particles, some charge will be transferred from left to right. Particles become charged and EDLs are formed close to their respective surfaces, leading to a potential jump $\Delta \Psi_{E D L}$ (salty) opposite to the Donnan potential. The process stops when particles become equipotential, that is $\Delta \Psi_{D}^{+}$(salty) $=-\Delta \Psi_{E D L}$ (salty). The potential profile is represented in Fig. 1b-stage 1. Note that the potential profile in this case differs from the previous stage in the vicinity of the core surface, where an EDL is created.

Next we disconnect the external circuit and change the solution to a more diluted ("fresh" hereafter) one $(20 \mathrm{mM} \mathrm{NaCl}$ 
solution in this case). Both Donnan potentials increase in absolute value and on the other hand, since the charge on the particle surfaces is fixed, also the potential jump at the particle/solution interface $\Delta \Psi_{E D L}$ increases in absolute value, as a consequence of the double layer expansion. ${ }^{20}$ Being the increase of the latter of smaller significance, the exchange to a fresh solution results in a net increase of the surface potential, as shown in Fig. 1b-stage 2. The surface potential becomes $\Psi_{S}^{ \pm}=\Delta \Psi_{D}^{ \pm}$(fresh) $+\Delta \Psi_{E D L}^{ \pm}($fresh $)$, and the potential difference is now $V=\left(\Delta \Psi_{D}^{+}(\right.$fresh $)+\Delta \Psi_{E D L}^{+}($fresh $\left.)\right)-$ $\left(\Delta \Psi_{D}^{-}(\right.$fresh $)+\Delta \Psi_{E D L}^{-}($fresh $\left.)\right)$. This results in a gain of stored energy.

In the next step we reconnect again both particles. Since there is a potential gradient directed to the right, charge is transferred in this direction, leading to a current flow in the external circuit. The potential profile is again modified only close to the core surface, in accordance to the change of surface charge. The process stops when particles become equipotential, and this occurs when $\Delta \Psi_{D}^{+}$(fresh) $=-\Delta \Psi_{E D L}($ fresh $)$, as shown in Fig. 1b-stage 3.

Finally, in open circuit configuration, we exchange fresh by salty water, and both potential rises $\Delta \Psi_{D}^{ \pm}$and $\Delta \Psi_{E D L}$ decrease, reaching the situation in Fig. 1b-stage 4. In this case, the potential profile in the vicinity of the electrode does not change with respect to the previous stage, but it is shifted following the change of the Donnan potential at the polyelectrolyte shell. Once the circuit is closed again, the potential difference drives a charge back to the left electrode until stage 1 is reached.

This process is similar to that of CDP, but the potential difference that can be generated between electrodes made of these particles has a different origin. In CDP, membranes are in contact with solutions of different salinities at each side, and hence, with different Donnan potentials. Hence, a membrane potential is generated and this is the surface potential on the electrode. ${ }^{11,25,26}$ In the process of Fig. 1, polyelectrolyte layers are attached to the particles, and hence, the surface potentials are directly the Donnan potentials.

The energy that can be extracted per unit area of interface in such cycle is

$$
\begin{aligned}
& E=\int_{\sigma_{2}}^{\sigma_{3}}\left(\Psi^{+}(\text {fresh })-\Psi^{-}(\text {fresh })\right) d \sigma+ \\
& \int_{\sigma_{4}}^{\sigma_{1}}\left(\Psi^{+}(\text {salty })-\Psi^{-}(\text {salty })\right) d \sigma
\end{aligned}
$$

where $\sigma_{i}$ is the surface charge density at stage " $i$ " of Fig. 1.

The electric potential $\Psi$ follows Poisson equation:

$$
\nabla^{2} \Psi(\mathbf{r})=-\frac{\rho_{\text {elec }}(r)}{\varepsilon_{0} \varepsilon}
$$

where $\rho_{\text {elec }}$ is the volume charge density at position $\mathbf{r}$ in the solution, and $\varepsilon_{0}$ and $\varepsilon$ are the electric permittivity of vacuum and the relative permittivity of the solvent, respectively.
The charge density in the conducting core is set to zero at the beginning. We assume that the polyelectrolyte shell is uniformly charged and that it is permeable to the solution. Hence, in this region the charge density is the sum of the charge density of the polyelectrolyte $\left(\rho_{p o l}\right)$ and that of the solution. Finally, outside the polyelectrolyte layer, the charge comes from free ions in the solution. The carbon layer is modeled as a swarm of spherical particles ${ }^{23}$ with radius $20 \mathrm{~nm}$ and average distance (equivalent to average pore radius) equal to $20 \mathrm{~nm}$. We assume that at least the outermost layers of carbon particles are coated with the polyelectrolyte, and the problem of potential distribution in the pore is solved for a single particle taking into consideration the possible double layer overlap between neighbor particles. Eq. 2 must be solved separately in three regions:

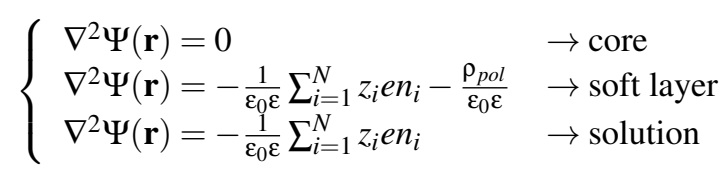

where $z_{i}$ and $n_{i}$ are, respectively, the valency and number concentration of ion of type $i$ and $e$ is the electron charge. The ionic charge density, $n_{i}$, follows the Boltzmann distribution:

$$
n_{i}=n_{i}^{\text {bulk }} \exp \left(-\frac{z_{i} e \Psi}{k_{B} T}\right)
$$

In eq. $4, n_{i}^{\text {bulk }}$ is the density of ions of type $i$ in the bulk, $k_{B}$ the Boltzmann constant and $T$ the absolute temperature. Spherical symmetry is assumed for solving the problem, so the quantities of interest will depend only on the radial coordinate $r$. The problem is totally defined by the boundary conditions at the interfaces:

$$
\left.\begin{aligned}
& \frac{\mathrm{d} \Psi}{\mathrm{d} r} \\
& \frac{\mathrm{d} \Psi}{\mathrm{d} r}
\end{aligned}\right|_{\text {bulk }} ^{\text {core surface }}=0
$$

In the boundary between the polyelectrolyte layer and the solution, the continuity of electric potential and its derivative must be satisfied:

$$
\begin{gathered}
\left.\Psi\right|_{S C^{+}}=\left.\Psi\right|_{S C^{-}} \\
\left.\frac{\mathrm{d} \Psi}{\mathrm{d} r}\right|_{S C^{+}}=\left.\frac{\mathrm{d} \Psi}{\mathrm{d} r}\right|_{S C^{-}}
\end{gathered}
$$

where $\mathrm{SC}^{-}\left(\mathrm{SC}^{+}\right)$indicates the inner (outer sides of the boundary between the polyelectrolyte layer and the solution.

For the solution of the above equations and boundary conditions, we used the routine BVP4C in Matlab ${ }^{\circledR}$ software. From the solution, the potential profiles in Fig. 1 can be obtained, whereas the surface charge on the core for a given potential follows from eq. 5. Furthermore, if the polyelectrolyte 
layer is thick enough, the potential reaches a uniform value different from that at the bulk solution, namely the Donnan potential. This decays to zero outside the polyelectrolyte layer in a distance of the order of the EDL thickness.

An example of a whole theoretical cycle is shown in Fig. 2. Note that the area of the shaded region $\left(3.1 \mathrm{~J} / \mathrm{m}^{2}\right.$ in this case) is the energy density that can be extracted in this process.

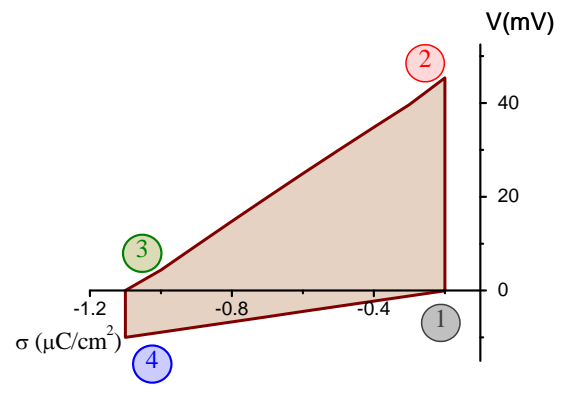

Fig. 2 Voltage between electrodes built with particles as in Fig. 1a as a function of the surface charge density for 20 and $500 \mathrm{mM} \mathrm{NaCl}$ solutions. The polyelectrolyte charge densities used were:

$\rho_{\text {pol }}=6 \times 10^{6}$ and $-3.8 \times 10^{6} \mathrm{C} / \mathrm{m}^{3}$ for the PDADMAC- and PSS-coated electrodes, respectively. The stages of the process are numbered as in Fig. 1b.

The feasibility of the method is subject to the success in covering activated carbon particles with a polyelectrolyte shell. We have tested two polyelectrolytes. One of them is the anionic poly(sodium 4-styrenesulfonate) (PSS) with typical molecular weight 70000, and the other one is the cationic poly(diallyldimethylammonium chloride) with typical molecular weight 100000 - 200000 (PDADMAC), both from Sigma Aldrich (USA). In order to investigate the capability of adsorption of these polyelectrolytes onto activated carbon particles, we determined the electrophoretic mobility of suspensions of such particles. For this purpose, we used a Malvern Nano ZS (Malvern Instruments, UK). We prepared three suspensions of the activated carbon particles both bare and immersed in aqueous 0.02 M PSS and 0.02 M PDADMAC solutions. Carbon particles were kindly supplied by Mast Carbon Ltd (UK). In Table 1 we present the electrophoretic mobility $\left(u_{e}\right)$ for both bare particles and those coated with the polyelectrolytes.

The change in mobility for treated particles indicates the presence of a polymer layer adhered to the surface. ${ }^{28}$ In particular, the larger negative mobility value for PSS-treated particles as compared to bare ones indicates that a negatively charged polymer layer is indeed coating the particle. Similarly, the change of sign in the case of PDADMAC-treated particles shows that the carbon is also coated by a positively charged layer screening the natural charge of the bare particle.
In fact, using the general electrokinetic model for soft particles described in, ${ }^{29}$ we can estimate that the polyelectrolyte charge densities are $+6 \times 10^{6}$ (PDADMAC) and $-3.8 \times 10^{6}$ (PSS) $\mathrm{C} / \mathrm{m}^{3}$. Similar values were obtained for these polyelectrolytes in a previous work where dielectric dispersion and dynamic electrophoresis data in oxide suspensions were used. ${ }^{30}$

Table 1 Electrophoretic mobility of activated carbon particles

\begin{tabular}{ll}
\hline Coating & $u_{e}$ \\
& $10^{-8} \mathrm{~m}^{2} \mathrm{~V}^{-1} \mathrm{~s}^{-1}$ \\
\hline Bare particles & -3.1 \\
PSS & -4.8 \\
PDACMAC & +8.5 \\
\hline
\end{tabular}

Having proved that it is possible to functionalize the carbon particles and convert them into "soft" particles, we performed the same procedure with commercial graphite-supported carbon films manufactured by Voltea B.V. (The Netherlands). Films were contacted with $0.02 \mathrm{M}$ PSS and 0.02 M PDADMAC solutions, respectively, while magnetically stirring the solutions, during $12 \mathrm{~h}$. They were placed in a parallel plate cell (Fig. 3) in the form of $2 \mathrm{~cm}$ diameter disks separated by $0.5 \mathrm{~mm}$. The electrodes are connected to a voltmeter and the open circuit voltage, or the potential at the extremes of a load resistor are determined. $500 \mathrm{mM}$ and $20 \mathrm{mM} \mathrm{NaCl}$ solutions are forced through the cell by performing regular exchanges, with typical durations $15 \mathrm{~s}$ salt water and $30 \mathrm{~s}$ in fresh water.

The resulting open circuit voltage between the electrodes as a function of time is represented in Fig. 4. We can see that it is clearly controlled by the salinity of the solution in contact with the electrodes, changing by around $70 \mathrm{mV}$ when sea and fresh waters are exchanged. In this figure we can also notice that after a fast increase of the voltage difference between the electrodes, there is a slow decrease, due to the leakage present in the device.

The cell voltage oscillations observed in Fig. 4 when fresh and sea waters are alternatively pumped between the electrodes are $62.3 \pm 0.3 \mathrm{mV}$ on the average. It is interesting that such oscillations can be obtained using the polymer charge densities obtained from mobility data as input for our model, as described above. As shown in Fig. 2, the voltage oscillations are almost identical to those experimentally obtained. Note that the maximum (thermodynamically allowed) cell voltage $(160 \mathrm{mV})$ is not reached and this can be ascribed to the fact that the polyelectrolyte layer is not dense enough, particularly in the case of PSS. It can be estimated that $\rho_{p o l}$ should be close to $10^{7} \mathrm{C} / \mathrm{m}^{3}$ in both coatings to achieve that goal. This gives us clues regarding the estimation of the energy efficiency $\eta$ of the method, as discussed below.

Obtaining energy from the soft carbon electrodes requires connection to the external load resistor. The cycle we implemented included the following steps (Fig. 5): short-circuit of 
a)

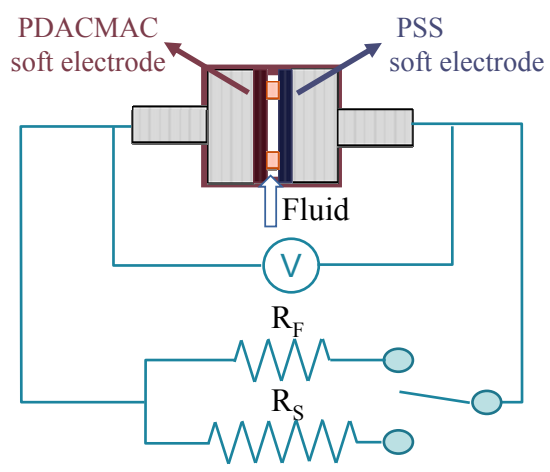

b)

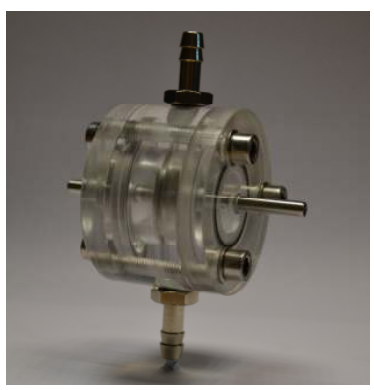

Fig. 3 a) Schematic representation of the circuit with the cell. The resistance is $R_{S}=10 \Omega$ in salty water and $R_{F}=60 \Omega$ in fresh water. b) Picture of the actual cell.

the electrodes in sea water; open circuit and fresh water in (25 $\mathrm{s}$ ); connection of the cell with the external resistor (measured current $0.75 \mathrm{~mA}$ at most) $(55 \mathrm{~s})$; open-circuit and sea water in $(25 \mathrm{~s})$; connection with the external load (55 s), current ( $0.5 \mathrm{~mA}$ maximum) flows in opposite direction. The voltage and current were continuously recorded, and from this the instantaneous power can be computed; alternatively, in order to make it easier the comparison with the theoretical cycles like that in Fig. 2, the instantaneous charge can be computed by integration of the intensity vs. time data, and the energy at each time calculated as the product of the measured voltage and the calculated charge.

From the linear fitting of the energy vs. time data, a value of average power can hence be obtained, as illustrated in Fig. $5 \mathrm{~d}$. We did not perform a systematic study of the timing optimization, but with the conditions described the power was $12.1 \pm 0.1 \mathrm{~mW} / \mathrm{m}^{2}$ (referred to electrode apparent area). Note that this power corresponds to an energy per cycle of $0.58 \mathrm{~mJ}$, taking $160 \mathrm{~s}$ as the cycle duration. In order to compare this figure with the theoretical prediction given in Fig. 2, it is necessary to carry out an estimation of the effective interfacial area of the electrode for a given geometrical area. In our case,

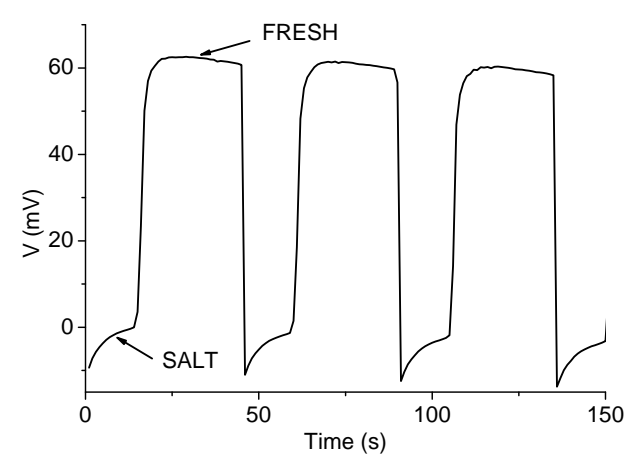

Fig. 4 Open circuit voltage measurements as a function of time.

superimposing the experimental voltage drop in step 2-3 with theoretical predictions would yield a surface charge density variation of $0.3 \mu \mathrm{C} / \mathrm{cm}^{2}$. Since the charge transferred in that step is $18 \mathrm{mC}$ (integration of the current-time data in Fig. 5b), we can conclude that active area would be $6 \mathrm{~m}^{2}$. Then the two cycles (measured and calculated) can be superimposed at the same scale, as shown in Fig. 5c. The shaded area would correspond to the portion of the cycle actually swept experimentally, and its value is $0.73 \mathrm{~mJ}$. From this, we conclude that there is a reasonable agreement between theoretical and experimental energy evaluations per cycle.

The efficiency $\eta$ can first of all be calculated as the so-called fuel efficiency, regarding relating the energy produced to the free energy of mixing (per unit volume) $\Delta g=\Delta G / V$, of equal volumes of salty and fresh solutions. In terms of power:

$$
\eta_{f u e l}=\frac{<V I>}{\Delta g J}
$$

where $\langle V I\rangle$ is the average power, and $J$ is the flow rate of solutions through the cell $(1 \mathrm{~mL} / \mathrm{s})$. In our case, $\eta_{\text {fuel }}$ is around $0.5 \%$, comparable to values reported for CDP techniques. ${ }^{25} \mathrm{~A}$ more practical approach is the calculation of the efficiency by comparing the area of the experimental cycle and of the ideal one without leakage and internal resistance: in such case, we obtain 36\% (see Fig. 5c).

Summarizing, the principle of extracting capacitive energy is used, taking advantage of the dependence of the Donnan potential with the salinity of the solution, in a similar way to CDP. The main difference is that the membranes are replaced by shells of polyelectrolytes, and that there is no space between shell and electrode surface, meaning that the potential difference between the electrodes is not related to the membrane potential but to the Donnan one. Hence, polyelectrolyte shells are a possible, cheaper alternative to membranes. Further research can shed light about the different behaviours of both methods concerning cleaning procedures, fouling or bio- 
fouling problems, as well as the differences in the kinetics of each of them.

\section{Acknowledgements}

The research leading to these results received funding from the European Union 7th Framework Programme (FP7/20072013) under agreement No. 256868. Further financial supports from Junta de Andalucía, project PE2012-FQM694, and Ministerio de Economía y Competitividad (Spain), project FIS2013-47666-C3-1-R, are also gratefully acknowledged.

\section{References}

1 R. E. Pattle, Nature, 1954, 174, 660-660.

2 R. S. Norman, Science, 1974, 186, 350-352.

3 R. E. Lacey, Ocean Eng., 1980, 7, 1-47.

4 J. W. Post, J. Veerman, H. V. M. Hamelers, G. J. W. Euverink, S. J. Metz, K. Nymeijer and C. J. N. Buisman, J. Membr. Sci., 2007, 288, 218-230.

5 T. Thorsen and T. Holt, J. Membr. Sci., 2009, 335, 103-110.

6 P. Dlugolecki, A. Gambier, K. Nijmeijer and M. Wessling, Environ. Sci. Technol., 2009, 43, 6888-6894.

7 Y. Kim and B. E. Logan, Environ. Sci. Technol., 2011, 45, 5834-5839.

8 A. Seppala and M. J. Lampinen, J. Membr. Sci., 1999, 161, 115-138.

9 J. Veerman, M. Saakes, S. J. Metz and G. J. Harmsen, Chem. Eng. J., 2011, 166, 256-268.

10 F. La Mantia, M. Pasta, H. D. Deshazer, B. E. Logan and Y. Cui, Nano Letters, 2011, 11, $1810-1813$.

11 M. Bijmans, O. Burheim, M. Bryjak, A. Delgado, P. Hack, F. Mantegazza, S. Tenisson and H. Hamelers, Energy Procedia, 2012, 20, 108 - 115

12 G. L. Wick and W. R. Schmitt, Mar. Technol. Soc. J., 1977, 11, 16-21.
13 S. Loeb, Science, 1975, 189, 654-655.

14 J. N. Weinstein and F. B. Leitz, Science, 1976, 191, 557-559.

15 K. Gerstandt, K. V. Peinemann, S. E. Skilhagen, T. Thorsen and T. Holt, Desalination, 2008, 224, 64-70.

16 A. Danilidis, R. Herber and D. A. Vermaas, Applied Energy, 2014, 119, 257-265.

17 W. Guo, L. Cao, J. Xia, F.-Q. Nie, W. Ma, J. Xue, Y. Song, D. Zhu, Y. Wang and L. Jiang, Adv. Funct. Mater, 2010, 20, 1339-1344.

18 A. Siria, P. Poncharal, A.-L. Biance, R. Fulcrand, X. Blase, S. T. Purcell and L. Bocquet, Nature, 2013, 494, 455-458.

19 M. Ye, M. Pasta, X. Xie, Y. Cui and C. S. Criddle, Energy Environ. Sci., 2014, 7, 2295-2300.

20 D. Brogioli, Phys. Rev. Lett., 2009, 103, 058501.

21 D. Brogioli, R. Zhao and P. M. Biesheuvel, Energy Environ. Sci., 2011, 4, $772-777$.

22 R. A. Rica, D. Brogioli, R. Ziano, D. Salerno and F. Mantegazza, J. Phys. Chem. C, 2012, 116, 16934-16938.

23 M. Jiménez, M. Fernández, S. Ahualli, G. Iglesias and A. Delgado, J. Colloid Interface Sci., 2013, 402, 340 - 349.

24 G. R. Iglesias, M. M. Fernández, S. Ahualli, M. L. Jiménez, O. P. Kozynchenko and A. V. Delgado, J Power Sources, 2014, 261, 371 377.

25 B. B. Sales, M. Saakes, J. W. Post, C. J. N. Buisman, P. M. Biesheuvel and H. V. M. Hamelers, Environ. Sci. Technol., 2010, 44, 5661-5665.

26 F. Liu, O. Schaetzle, B. B. Sales, M. Saakes, C. J. N. Buisman and H. V. M. Hamelers, Energy Environ. Sci., 2012, 5, 8642-8650.

27 M. C. Hatzell, R. D. Cusick and B. E. Logan, Energy Environ. Sci., 2014, 7, 1159-1165.

28 H. Ohshima, Journal of Colloid and Interface Science, 2000, 228, 190193.

29 S. Ahualli, M. L. Jiménez, F. Carrique and A. V. Delgado, Langmuir, 2009, 25, 1986-1997.

30 S. Ahualli, Ph.D. thesis, University of Granada, 2009. 

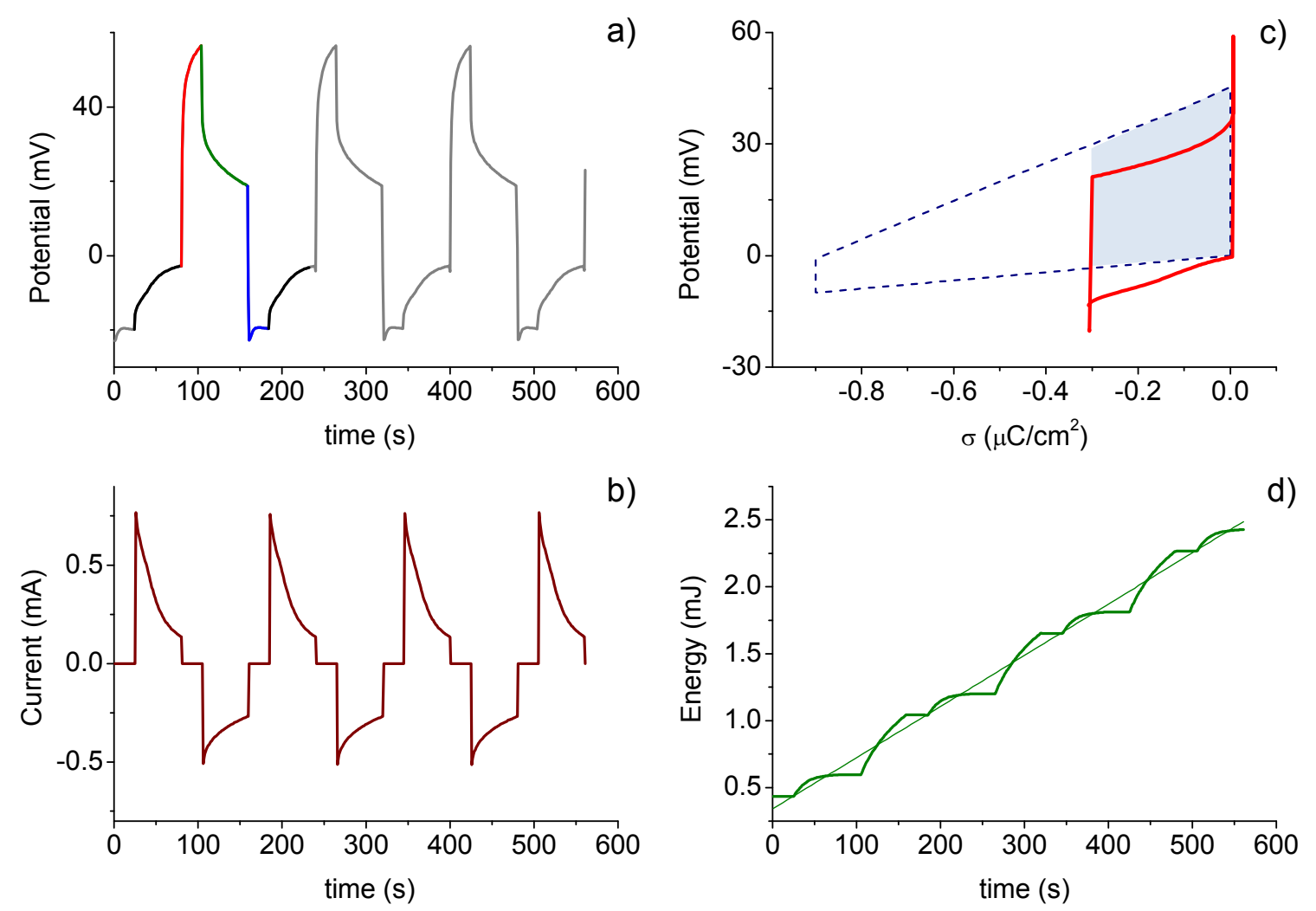

Fig. 5 Voltage (a) and current (b) measurements in the cell as a function of time. c) Experimental (solid red line) and theoretical (blue dashed line) cell voltage vs. charge density cycles; the shaded area corresponds to the portion of the calculated cycle swept by the experiment. d) Accumulated energy as a function of time. The slope of the fitted straight line is the power. In this case, $12.1 \mathrm{~mW} / \mathrm{m}^{2}$ of electrode. 